\title{
Momentum distribution and non-local high order correlation functions of 1D strongly interacting Bose gas
}

\author{
EJKP Nandani ${ }^{1,2,3}$ and Xi-Wen Guan ${ }^{1,4, \text { * }}$ \\ ${ }^{1}$ Wuhan Institute of Physics and Mathematics, Chinese Academy of Sciences, Wuhan 430071, China. \\ ${ }^{2}$ University of Chinese Academy of Sciences, Beijing 100049, China. \\ ${ }^{3}$ Department of Mathematics, University of Ruhuna, Matara 81000, Sri Lanka. \\ ${ }^{4}$ Department of Theoretical Physics, Research School of Physics and Engineering, \\ Australian National University, Canberra ACT 0200, Australia.
}

(Dated: June 13, 2021)

\begin{abstract}
The Lieb-Liniger model is a prototypical integrable model and has been turned into the benchmark physics in theoretical and numerical investigations of low dimensional quantum systems. In this note, we present various methods for calculating local and nonlocal $M$-particle correlation functions, momentum distribution and static structure factor. In particular, using the Bethe ansatz wave function of the strong coupling Lieb-Liniger model, we analytically calculate twopoint correlation function, the large moment tail of momentum distribution and static structure factor of the model in terms of the fractional statistical parameter $\alpha=1-2 / \gamma$, where $\gamma$ is the dimensionless interaction strength. We also discuss the Tan's adiabatic relation and other universal relations for the strongly repulsive Lieb-Liniger model in term of the fractional statistical parameter.

Keywords: Correlation function, Momentum distributions, Structure Factor, Contact
\end{abstract}

\section{INTRODUCTION}

The Bethe ansatz, which was introduced in 1931 by Hans Bethe, has become a powerful method to obtain exact solutions of one-dimensional (1D) quantum many-body systems. In 1963, Lieb and Liniger [1] solved the 1D many-particle problem of $\delta$-function interacting bosons by the Bethe's hypothesis. The ground state, the momentum, and elementary excitations were obtained for this model by using the Lieb-Liniger solution. In this context, a significant step was made on the discovery of the grand canonical description of this Lieb-Liniger model by Yang and Yang in 1969 [2]. Now, this grand canonical approach is called Yang-Yang thermodynamic method. The Yang-Yang thermodynamics of the Lieb-Liniger Bose gas provides benchmark understanding of quantum statistics, thermodynamics and quantum critical phenomena in many-body physics, see a review [3, 4]. In the context of ultracold atoms, the 1D Bose gas with a repulsive short-range interaction characterized by a tunable coupling constant exhibits rich many-body properties. This model thus becomes an ideal test ground to explore fundamental many-body phenomena ranging from equilibrium to nonequilibrium physics in the experiment [5-10].

Despite Lieb-Liniger is arguably the simplest integrable model, the calculation of correlation functions is extremely challenging due to the complexity of the Bethe ansatz many-body wave function of the model. The study of correlation functions has long been being an important theme in the physics of ultracold quantum gases since they provide information of quantum many-particle interference and coherence beyond the solely spectra of the systems. Therefore, there has been much theoretical and experimental interest in the local, non-local, and dynamical correlation functions at zero and finite temperatures via numerous methods based on exactly solvable models, see [11 22].

For sufficiently strong interaction and sufficiently low density, the 1D Lieb-Liniger gas enters the Tonks-Girardeau (TG) regime, in which bosons behave like impenetrable particles (hard-core bosons). Such impenetrable bosons behave mostly like the free fermions that build up the Girardeau's Bose-Fermi mapping [23]. In fact, the 1D Lieb-Liniger Bose gas with the interacting strength $c_{\mathrm{B}}$ can map onto the fully-polarized fermions with a p-wave interaction of strength $c_{\mathrm{F}}=1 / c_{\mathrm{B}}$ [24]. As a result of the Bose-Fermi mapping, the energy spectra of the Bose and corresponding Fermi system are identical at the TG regime. The observables that can be given in terms of the local density are identical for both systems, such as dynamical density-density correlation function, see [25]. However, this mapping for the off-diagonal correlation function does not like to be true, for example, momentum distribution. The Bose-Fermi mapping has tremendous applications in the study of strongly interacting quantum gases of ultracold atoms. An expansion of the reduced density matrices for $1 D$ bosons has been first derived by Lenard [26, 27] using bose-fermi mapping at Tonks Girardeau regime. After that, there were superb findings in the literature, for example, [28 35]. 
In the context of correlation functions, the Fourier coefficients have an important physical significance. By taking Fourier transform of the one- and two-particle correlation functions, the momentum distribution and the static structure factor of the system can be obtained, respectively. The first few terms of the short-range series expansion of the one-particle correlation function $g_{1}(x)=1+\sum_{i=0}^{\infty} c_{i}|n x|^{i}$ have been calculated in [28, [36], and 37], where $c_{1}=0, c_{2}=1 / 2\left(e(\gamma)-\gamma e^{\prime}(\gamma)\right)$ and $c_{3}=\gamma^{2} e^{\prime}(\gamma) / 12$, etc. A further study on the connection between the non-local one-body and local three-body correlation functions was presented in [38]. The systems described by the Hamiltonian with short distance interaction ( $\delta$-function type) show singular behaviour of the wave function when two or more colliding particles coincide. Consequently, the large momentum tail of the momentum distribution has a universal power-law decay, i.e. $w(p) \sim C / p^{4}$ where $C$ is an extensive quantity called Tan's contact [39 41]. Beside the power law tail of the momentum distribution, the contact characterizes many universal properties of the systems that are independent of the details of the contact interaction and the dimensionality [42], such as the energy adiabatic relation, the universal connection to the structure factor and the pressure relation. The contact measuring the correlation of such systems of this kind in short distance limit is proportional to the probability of finding two atoms at zero separation [39 41], see more studies on the contact in [43 45]. The contact can be computed from the free energy of the system via the Helmann-Feynmann theorem [46], or from the prefactor of the high-frequency power-law decay of the single particle correlator [47], or by the operator product expansion field theory method [48], etc. Very recently, p-wave contacts were found to exhibit universal relations in the ultracold atomic systems with a p-wave interaction [49 51]. In this scenario, the momentum distribution, s-wave and pwave contact relations have been attracted a great deal of attention from theory and experiment [36, 37, 42, 46, 52 56].

On the other hand, the static structure factor contains information about the static correlation properties of a system and directly relates to the pair distribution function or the normalized density-density correlator or spin-spin correlator [25]. In the low-momentum region and the infinite repulsive regime, hydrodynamic theory predicts that the static structure factor should behave linearly with momentum, $k[42$. The static structure factor at small momentum $k$ is related to the sound velocity, i.e. $\left(v_{s}\right)$ by $S(k)=\hbar|k| /\left(2 m v_{s}\right)[12,21,22,25$, , 42, 57]. For high momentum, the structure factor always converges to 1 [42]. The Fourier transform of the time-dependent density-density correlation function is known as the dynamical structure factor [21, 58, 59], and expresses the probability of excitation by an infinitesimal perturbation.

The duality between interacting spinless bosons and fermions in 1D [24, 25, 32] can be adopted to the framework of the generalized fractional statistics, which interpolate continuously between bosons and fermions. In fact, while three dimensions particles can be only bosons or fermions, in lower dimensionality it has been recognized that particles with fractional statistics intermediate between bosons and fermions [60 66] due to the transmission between dynamical interaction and statistical interaction. The $1 D$ strongly repulsive Bose gas at low temperatures is equivalent to a gas of ideal particles obeying the non-mutual generalized exclusion statistics (GES) with a statistical parameter $\alpha=1-2 / \gamma$, where $\gamma$ is the dimensionless interaction strength [66]. Using such a mapping, i.e. the quasimomenta of $N$ strongly interacting bosons map to the momenta of $N$ free fermions via $k_{i} \approx \alpha k_{i}^{F}$ with $i=1, \ldots, N[66]$. By using the fractional statistical parameter $\alpha$, the $M$-body local correlation functions and non-local correlation functions in terms of the wave function of $M$ bosons at zero collision energy and zero total momentum have been computed analytically up to next-to-leading order [19]. In this paper, we analytically calculate various correlation functions in terms of the statistical parameter $\alpha$. In particular, we calculate the two-point correlation function, the power-law tail of momentum distribution and discuss the structure factor of $1 D$ Lie-Liniger model of ultracold atoms with finitely strong interaction strength. For Tonks gas, i.e. $\alpha=1$, our results are equivalent to that of the Tonks-Girardeau gas.

The paper is organized as follows: In sections I and II, we briefly introduce the Lieb-Liniger model and recall some results of higher order local correlations for the 1D interacting bosons. In section III, we present the derivation of the nonlocal $2 M$-point correlation function. In Section IV, we prove the power-law decay of the momentum distribution of the 1D interacting bosons at large momenta. The large momentum tail of the momentum distribution determines Tan's contact in term of the fractional statistical parameter $\alpha$. In this section, we also discuss the static structure factor. The last section presents our conclusion. 


\section{MODEL OF INTEREST}

A model of $1 D$ spinless $N$ bosons with $\delta$-function interaction is described by the Hamiltonian

$$
H_{N}=\frac{\hbar^{2}}{2 m}\left(\sum_{j=1}^{N}-\partial_{x_{j}}^{2}+2 c \sum_{1 \leq j<i \leq N} \delta\left(x_{j}-x_{i}\right)\right)
$$

where $m$ is the mass of bosons and $c=-2 / a_{1 D}$ is a coupling constant. Here, $a_{1 D}$ is the $1 \mathrm{D}$ scattering length and $\hbar$ is the Plank constant. The positive and negative $c$ values correspond to the repulsive and attractive interactions, respectively, and $c=0$ for free particles.

With the periodic boundary condition, $\psi\left(0, x_{2}, \ldots, x_{N}\right)=\psi\left(x_{2}, \ldots, x_{N}, L\right)$, in the domain $0 \leq x_{1} \leq x_{2} \leq \ldots \leq$ $x_{N} \leq L$, the Betha ansatz wave function can be written as [1]

$$
\psi\left(x_{1}, \ldots, x_{N}\right)=\sum_{P}(-1)^{P}\left[\prod_{1 \leq i<j \leq N}\left(\frac{\mathrm{i} c+k_{P_{i}}-k_{P_{j}}}{\mathrm{i} c-k_{P_{i}}+k_{P_{j}}}\right)^{1 / 2}\right] e^{\mathrm{i} \sum_{j=1}^{N} k_{P_{j}} x_{j}},
$$

where $(-1)^{P}$ is parity for the permutations and the sum runs over $N$ ! permutations $P$ with respect to quasi-momentum $\left\{k_{1}, k_{2}, \ldots, k_{N}\right\}$. After expanding the amplitude of the wave function, we have

$$
\psi\left(x_{1}, \ldots, x_{N}\right)= \begin{cases}\mathcal{A}(c) \sum_{P}(-1)^{P} e^{\mathrm{i} \sum_{j=1}^{N} k_{P_{j}} x_{j}}, & \text { for strong coupling } \\ N !-\frac{c}{2} Y\left(x_{1}, \ldots, x_{N}\right), & \text { for weak coupling }\end{cases}
$$

where $\mathcal{A}(c)=\left(1+\frac{1}{c} \sum_{j=1}^{N}(2 j-N-1) \partial_{x_{j}}\right)$ and $Y\left(x_{1}, \ldots, x_{N}\right)=\sum_{P}\left(\sqrt{\frac{L}{2}} \sum_{i<j} F\left(q_{P_{i}}-q_{P_{j}}\right)+\sqrt{\frac{2}{L}} \sum_{j=1}^{N} q_{P_{j}} x_{j}\right)^{2}$, here $F\left(q_{i}-q_{j}\right)=\left(q_{i}-q_{j}\right)^{-1}$ and $q_{j}=k_{j} \sqrt{L / 2 c}$. The derivation of the wave function for weak coupling can be seen in Appendix A, also see the case in the $1 \mathrm{D}$ p-wave fermions in [56]. Moreover, the normalization factor of the wave function is defined by $\mathcal{N}^{2}=\int_{0}^{L} d x_{1} \ldots d x_{N}\left|\psi\left(x_{1}, x_{2}, \ldots, x_{N}\right)\right|^{2}$. Further, the wave function is continuous whenever two particles close to each other, and the discontinuity in the derivative of the wave function is $2 c$ when two particles overlap. These are known as boundary conditions of the wave function with $\delta$ potential [1]. For a given set of quasi-momenta $\left\{k_{j}\right\}$, the total momentum and the energy of the system are $\mathcal{P}=\sum_{j=1}^{N} k_{j}$ and $E=\sum_{j=1}^{N} k_{j}^{2}$, respectively.

For a finite large interaction strength, the quasimomenta of the bosons $k_{1}, k_{2}, \ldots, k_{N}$ deviate from pure Fermi statistics, they obey the non-mutual general exclusive statistics (GES) 60, 61, 66]. The deviation from Fermi statistics is determined by the non-mutual GES parameter $\alpha=1-2 / \gamma$ [66]. If the total momentum $k_{1}+\cdots+k_{N}=0$, we have the following relation:

$$
k_{j}=k_{j}^{F} \alpha+O\left(c^{-2}\right),
$$

where $k_{j}^{F}=2 \pi \mathcal{I}_{j} / L$ and the $\mathcal{I}_{j}$ 's are integers satisfying $\mathcal{I}_{1}<\mathcal{I}_{2}<\cdots<\mathcal{I}_{N}$. In fact, the equal spacing in momenta reveals a nature of non-mutual fractional statistics. Here, the wave function of $1 D$ interacting bosons can be given in terms of the GES $k_{j}=k_{j}^{F} \alpha$,

$$
\psi\left(x_{1}, \ldots, x_{N}\right)= \begin{cases}\left(1+\frac{\alpha}{c} \sum_{j=1}^{N}(2 j-N-1) \partial_{x_{j}^{F}}\right) \psi_{N}^{F}, & x_{j}=x_{j}^{F} / \alpha, \\ \left(1+(\alpha-1) \sum_{j=1}^{N} x_{j}^{F} \partial_{x_{j}^{F}}+\frac{1}{c} \sum_{j=1}^{N}(2 j-N-1) \partial_{x_{j}^{F}}\right) \psi_{N}^{F}, & x_{j} \sim x_{j}^{F},\end{cases}
$$

where $\psi_{N}^{F}=\sum_{P}(-1)^{P} \exp \left(\mathrm{i} \sum_{j=1}^{N} k_{P_{j}}^{F} x_{j}^{F}\right)$ is the wave function of $N$ free fermions. 


\section{LOCAL $M$-BODY CORRELATION FUNCTIONS}

We first would like to recall some results of local $M$-body correlation functions which have been studied [1] 22]. In particular, we discuss the results of the correlation functions which were recently derived by using the GES relation Eq. (4). It turns out that the GES is convenient to calculate the local $M$-body correlation functions.

\section{II.1 Strong coupling regime}

The local $M$-particle correlation is a measure of the probability of observing $M$ particles at the same position and defined by

$$
g_{M}=\frac{N !}{(N-M) !} \frac{\int_{0}^{L} \ldots \int_{0}^{L} d x_{M+1} \ldots d x_{N}\left|\psi\left(0, \ldots, 0, x_{M+1}, \ldots, x_{N}\right)\right|^{2}}{\int_{0}^{L} \ldots \int_{0}^{L} d x_{1} \ldots d x_{N}\left|\psi\left(x_{1}, \ldots, x_{N}\right)\right|^{2}} .
$$

In the strong coupling limit, one can expand the BA wave function, Eq. (3) in terms of the power of $1 / c$ from which the numerator and denominator can be calculated analytically [19]

$$
g_{M}=\frac{\left.N ! c^{-M(M-1)} \int_{0}^{L} \cdots \int_{0}^{L}\left|\triangle_{M}\left(\partial_{x}\right) \phi\left(x_{1}, \cdots, x_{N}\right)\right|_{x_{1}=\cdots=x_{M}=0}\right|^{2} d x_{M+1} \cdots d x_{N}+O\left(c^{-M(M-1)-2}\right)}{(N-M) !\left[1+\frac{2 N(N-1)}{c L}+O\left(c^{-2}\right)\right] \int_{0}^{L} \cdots \int_{0}^{L}\left|\phi\left(x_{1}, \ldots, x_{N}\right)\right|^{2} d x_{1} \cdots d x_{N}} .
$$

Here, $\Delta_{M}\left(\partial_{x}\right)=\prod_{1 \leq i<j \leq M}\left(\frac{\partial}{\partial x_{j}}-\frac{\partial}{\partial x_{i}}\right)$ is the Vandermonde determinant and $\phi\left(x_{1}, \ldots, x_{N}\right)=\sum_{P}(-1)^{P} e^{\mathrm{i} \sum_{j=1}^{N} k_{P j} x_{j}}$, for all $x$ 's. In this case, using the GES Eq. (4),$k_{i}=k_{i}^{F} \alpha$ and making a scaling change $x_{i}=x_{i}^{F} / \alpha$ for $i=1, \ldots, N$, thus we can write Eq. (7) as

$$
g_{M}=\frac{N !}{(N-M) !} \frac{\left.\frac{\alpha^{M^{2}-N}}{c^{M(M-1)}} \int_{0}^{L} \cdots \int_{0}^{L}\left|\Delta_{M}\left(\partial_{x^{F}}\right) \phi^{F}\left(x_{1}^{F}, \cdots, x_{N}^{F}\right)\right|_{x_{1}^{F}=\cdots=x_{M}^{F}=0}\right|^{2} d x_{M+1}^{F} \cdots d x_{N}^{F}}{\alpha^{-(N-1)} \int_{0}^{L} \cdots \int_{0}^{L}\left|\phi^{F}\left(x_{1}^{F}, \cdots, x_{N}^{F}\right)\right|^{2} d x_{1}^{F} \cdots d x_{N}^{F}},
$$

to the order $O\left(c^{-M(M-1)-2}\right)$. Here, $\phi^{F}\left(x_{1}^{F}, \cdots, x_{M}^{F}\right) \equiv \sum_{P}(-1)^{P} e^{\mathrm{i} \sum_{j=1}^{N} k_{P j}^{F} x_{j}^{F}}$. Since $\phi^{F}\left(x_{1}, \ldots, x_{N}\right)$ is a Slater determinant, by applying Wick's theorem and changing variable $k^{F}=2 \pi n z$, then the $M$-body correlation function reads

$$
g_{M}=M ! n^{M}(2 \pi / \gamma)^{M(M-1)} \alpha^{M^{2}-1} \int_{-\infty}^{\infty} \ldots \int_{-\infty}^{\infty} d z_{1} \cdots d z_{M}\left(\prod_{j=1}^{M} N\left(z_{j}\right)\right) \Delta_{M}^{2}(z)+O\left(\gamma^{-M(M-1)-2}\right),
$$

where the dimensionless parameter $\gamma=c / n$, the liner density is given by $n=N / L$ and $N(z)=$ $\left(1+\exp \left(\frac{\frac{\hbar^{2}}{2 m}(2 \pi n z)^{2}-\mu}{k_{B} T}\right)\right)^{-1}, T$ and $\mu$ are the temperature and the chemical potential of the 1D Bose gas. Calculating the multiple integrals in Eq. (9) by using the orthogonal polynomials in the random matrix theory [67], we can give the $M$-particle local correlation function as

$$
g_{M}=(M !)^{2} n^{M}(2 \pi / \gamma)^{M(M-1)} \alpha^{M^{2}-1} \prod_{i=0}^{M-1} h_{i}
$$

where $h_{i}$ 's are the norm-squares of the monic orthogonal polynomials satisfying $\int_{-\infty}^{\infty} P_{i}(z) P_{j}(z) N(z) d z=h_{i} \delta_{i j}$. Considering the distribution of particles at ground state and applying Sommerfeld expansion, we have general formulas for the higher order local correlation function in two temperature regimes [19]

$$
\frac{g_{M}}{n^{M}}= \begin{cases}\frac{\left(\prod_{j=1}^{M} j !\right)^{2} \alpha^{\left(M^{2}-1\right)}(\pi / \gamma)^{M(M-1)}}{\left(\prod_{j=1}^{M-1}(2 j-1) ! !\right)^{2}(2 M-1) ! !}\left\{1+\frac{1}{48} M^{2}\left(M^{2}-1\right)\left(\frac{T}{\pi T_{d}}\right)^{2}+O\left[\left(\frac{T}{T_{d}}\right)^{2}\right]\right\}, & T \ll T_{d}, \\ (M !)^{2} \alpha^{M^{2}-1}\left(\frac{T}{2 \gamma^{2} T_{d}}\right)^{\frac{M(M-1)}{2}} \prod_{j=0}^{M-1} j !, & T_{d} \ll T \ll \gamma^{2} T_{d},\end{cases}
$$


where $T_{d} \equiv \hbar^{2} n^{2} /\left(2 m k_{B}\right)$ is the quantum degeneracy temperature. In the above calculation, the statistical parameter $\alpha$ in the quasi-momenta of the hardcore bosons can be extracted as a factor giving the next-order corrections in the correlation function (11). In such a way, the integrant in the correlation function (8) reduces to the Vandermonde determinant with respect to the the wave function of the free fermions. Then the Wick's theorem and random matrix theory are used to obtain the explicit form of $M$-body correlation function (11). However, it would be extremely difficult to obtain higher order correction terms beyond the form of (11).

\section{II.2 Weak coupling regime}

For weak coupling case, one can easily use the wave function (3) to calculate the $M$-body correlation function

$$
\begin{aligned}
& g_{M}^{w}\left(y_{1}, \ldots, y_{M} ; x_{1}, \ldots, x_{M}\right) \\
&= \frac{N !}{(N-M) ! \mathcal{N}^{2}} \int_{0}^{L} \ldots \int_{0}^{L} d x_{M+1} \ldots d x_{N}\left(N !-\frac{c}{2} Y\left(y_{1}, \ldots, y_{M}, x_{M+1} \ldots, x_{N}\right)\right)^{*}\left(N !-\frac{c}{2} Y\left(x_{1}, \ldots, x_{N}\right)\right) \\
&= \frac{N !}{(N-M) ! \mathcal{N}^{2}}\left[N !^{2} L^{N-M}-N ! \frac{c}{2} \int_{0}^{L} \ldots \int_{0}^{L} d x_{M+1} \ldots d x_{N}\left[\left(Y\left(y_{1}, \ldots, y_{M}, x_{M+1} \ldots, x_{N}\right)\right)^{*}+Y\left(x_{1}, \ldots, x_{N}\right)\right]\right] \\
&+O\left(c^{2}\right) .(12)
\end{aligned}
$$

So, the $M$-particl local-correlation function becomes

$g_{M}^{w}(0, \ldots, 0 ; 0, \ldots, 0)=\frac{N !}{(N-M) ! \mathcal{N}^{2}}\left[N !^{2} L^{N-M}-N ! c \int_{0}^{L} \ldots \int_{0}^{L} d x_{M+1} \ldots d x_{N}\left[Y\left(0, \ldots, 0, x_{M+1}, \ldots, x_{N}\right)\right]\right]+O\left(c^{2}\right)$,

where $Y\left(0, \ldots, 0, x_{M+1}, \ldots, x_{N}\right)=\sum_{P}\left(\sqrt{\frac{L}{2}} \sum_{i<j}\left[F\left(q_{P_{i}}-q_{P_{j}}\right)\right]+\sqrt{\frac{2}{L}} \sum_{j=M+1}^{N} q_{P_{j}} x_{j}\right)^{2}$.

In fact, the Bogoliubov method is very convenient and useful for the study of the weak coupling Bose gas in any dimension. In this Bogoliubov approach, one consider a large fraction of particles in the lowest quantum state and excites a small fraction of particles out of the Bose-condensed particles with weakly repulsive interacting Bose gas. Therefore, in this regime, the field operator, $\hat{\Psi}$, can be considered as a sum of the condensate part, $\psi_{0}$, and a noncondensate part, $\psi^{\prime}$, such as $\hat{\Psi}=\psi_{0}+\psi^{\prime}$. The density of the system is $n=n_{0}+n^{\prime}$ where $n_{0}=\left\langle\psi_{0} \psi_{0}^{*}\right\rangle$ and $n^{\prime}=\left\langle\psi^{\prime \dagger} \psi^{\prime}\right\rangle$ represent the condensed particles density and non condensed particles density, respectively. For a uniform condensate, the ground state solution is $\psi_{0}=\sqrt{n_{0}} \exp (\mathrm{i} \mu t / \hbar)$, where the chemical potential is given by the relation $\mu=n_{0} g$, see a detailed study given in 13 .

In the weak coupling regime, $\gamma \ll 1$, the mean field interaction energy per particle proportional to $n g$, where $g=\hbar^{2} c / m$, and it is proper to define the correlation length, $l_{c}=\hbar / \sqrt{m n g}$. At temperature $T \ll T_{d}$, over a wide range of parameters $l_{c} \ll l_{\phi}$, we can have the condition $T / T_{d} \ll \sqrt{\gamma}[13]$. Here, $l_{\phi}$ is the phase coherence length and $T_{d}=\hbar^{2} n^{2} / 2 m$ is the quantum degeneracy temperature. In this case, the condensate fraction contains the contribution of excitations with momenta $k \leq k_{0} \ll l_{c}^{-1}$, and non-condensate parts provided excitations with $k \sim l_{c}^{-1}$ [13, 15]. In order to complete our discussion on the local $M$-body correlation, the necessary calculation of the correlation function in Appendix B. What below is a summary of the correlation functions based on the Bogoliubov approach.

The $M$-particle correlation function for $1 D$ bosons at weak coupling regime is given by

$$
g_{M}^{w}=\left\langle\left(\psi_{0}^{*}+\psi^{\prime \dagger}\right)^{M}\left(\psi_{0}+\psi^{\prime}\right)^{M}\right\rangle=n^{M}\left(1+\frac{M(M-1)}{n}\left(\left\langle\psi^{\prime \dagger} \psi^{\prime}\right\rangle+\left\langle\psi^{\prime} \psi^{\prime}\right\rangle\right)\right)
$$

dealing with the $M^{t h}$ order density $n^{M}=n^{M}-M n^{M-1}\left\langle\psi^{\prime \dagger} \psi^{\prime}\right\rangle+O\left(\left\langle\psi^{\prime \dagger} \psi^{\prime}\right\rangle\right)^{2}$. The Eq. (68) shows that $g_{M}^{w}$ depends on the normal density, $\left\langle\psi^{\prime \dagger} \psi^{\prime}\right\rangle$, and anomalous density, $\left\langle\psi^{\prime} \psi^{\prime}\right\rangle$, of the non-condensed part. Considering the form of the non-condensate part: $\psi^{\prime}=\sum_{k}\left(u_{k} \hat{b}_{k} e^{-\mathrm{i} \epsilon_{k} t / \hbar}-v_{k}^{*} \hat{b}_{k}^{\dagger} e^{\mathrm{i} \epsilon_{k} t / \hbar}\right), g_{M}^{w}$ can be converted into

$$
g_{M}^{w}=n^{M}\left(1+\frac{M(M-1)}{2 \pi n} \int_{-\infty}^{\infty} d k\left(\frac{E_{k}}{\epsilon_{k}}\left(N_{k}+1\right)-1\right)\right),
$$

by Bogoliubov transformation, where $\hat{b}_{k}, \hat{b}_{k}^{\dagger}$ are operators of excitations which obey the usual commutation relations, $\epsilon_{k}$ are their eigen energies, and $u_{k}, v_{k}$ their eigenfunctions. This result illustrates that $g_{M}^{w}$ depends on the occupation 
number of the excitation, $N_{k}$, excitation energy, $\epsilon_{k}$, and system energy $E_{k}$. In fact, $g_{M}^{w}$ varies with the temperature range since $N_{k}=0, \epsilon_{k} \approx \sqrt{E_{k}^{2}+2 g n_{0} E_{k}}$ at zero temperature and $N_{k} \neq 0, \epsilon_{k}=\hbar k v_{s}$ at non-zero temperature. Here, $v_{s} \approx \sqrt{n_{0} g / m}$ is the sound velocity and the distribution $N_{k}$ takes the form $\left(e^{\epsilon_{k} / T}-1\right)^{-1}$ for the temperature $T \ll \mu$ and $T \gg \mu$, respectively. However, $E_{k}=\hbar^{2} k^{2} / 2 m$ and $\mu \approx n_{0} g$ at low temperature.

This study followed the method developed by Gangardt and Shlyapnikov in [13]. The elementary excitation of the Bose-condensed system involves vacuum fluctuations and thermal fluctuations. At zero temperature, thermal fluctuations are suppressed and vacuum fluctuations dominate. This scenario becomes reversed at $\mu \ll T \ll \sqrt{\gamma} T_{d}$. However, both vacuum and thermal fluctuations create excitations at $T \ll \mu$.

According to these configurations, and after a length algebra, the $M$-particle correlation, Eq. (15) is given by

$$
\frac{g_{M}^{w}}{n^{M}}= \begin{cases}1-\frac{M(M-1)}{\pi} \sqrt{\gamma}, & T=0, \\ 1-\frac{M(M-1)}{\pi} \sqrt{\gamma}+\frac{M(M-1) T^{2} \pi}{48} \frac{\sqrt{\gamma}}{\left(\gamma T_{d}\right)^{2}}, & T \ll \mu, \\ 1+\frac{M(M-1) T}{4 \pi \sqrt{\gamma} T_{d}}, & \sqrt{\gamma} T_{d} \gg T \gg \mu .\end{cases}
$$

These results are not valid for $T \geq T_{d} \sqrt{\gamma}$, belonging to quantum decoherence regime. The detailed calculation is presented in Appendix B. This result has been reported in [13]. The study 2-body correlations was also reported in [14], while the 2-body, 3-body correlation functions at zero temperature were studied in [15].

\section{Non-local correlation functions}

The $M$-particle non-local correlation is a measure of the probability of observing $M$ particles at different points and defined by

$$
\begin{aligned}
& g_{M}\left(x_{1}, \ldots, x_{M} ; x_{1}^{\prime}, \ldots, x_{M}^{\prime}\right) \\
= & \frac{N !}{(N-M) !} \frac{\int_{0}^{L} \ldots \int_{0}^{L} d x_{M+1} \ldots d x_{N} \psi^{*}\left(x_{1}^{\prime}, \ldots, x_{M}^{\prime}, x_{M+1}, \ldots, x_{N}\right) \psi\left(x_{1}, \ldots, x_{M}, x_{M+1}, \ldots, x_{N}\right)}{\int_{0}^{L} \ldots \int_{0}^{L} d x_{1} \ldots d x_{N}\left|\psi\left(x_{1}, \ldots, x_{N}\right)\right|^{2}} \\
= & \frac{N !}{(N-M) !} \frac{\int_{0}^{L} \ldots \int_{0}^{L} d x_{M+1} \ldots d x_{N} \psi^{*}\left(0, \ldots, 0, x_{M+1}, \ldots, x_{N}\right) \psi\left(x_{1}-x_{1}^{\prime}, \ldots, x_{M}-x_{M}^{\prime}, x_{M+1}, \ldots, x_{N}\right)}{\int_{0}^{L} \ldots \int_{0}^{L} d x_{1} \ldots d x_{N}\left|\psi\left(x_{1}, \ldots, x_{N}\right)\right|^{2}} \\
= & g_{M}\left(x_{1}-x_{1}^{\prime}, \ldots, x_{M}-x_{M}^{\prime} ; 0, \ldots, 0\right) .
\end{aligned}
$$

The Galilean invariance is used in the above equation. The short distance non-local correlation functions $\left\langle\Psi^{\dagger}\left(x_{1}^{\prime}\right) \cdots \Psi^{\dagger}\left(x_{M}^{\prime}\right) \Psi\left(x_{M}\right) \cdots \Psi\left(x_{1}\right)\right\rangle$ of the strongly repulsive Bose gas was studied in terms of the wave function of $M$ bosons at zero collision energy and zero total momentum in [19].

\section{III.1 Strong coupling regime}

We first consider the non-local correlation function of strongly interacting $1 D$ bosons with $c=\infty$. Let's shorten our notation $g_{M}\left(x_{1}, \ldots, x_{M}\right)=g_{M}\left(x_{1}, \ldots, x_{M} ; 0, \ldots, 0\right)$. According to the Girardeau's Bose-Fermi mapping [23], we have $g_{M}^{B}\left(x_{1}, \ldots, x_{M}\right) \approx\left|g_{M}^{F}\left(x_{1}, \ldots, x_{M}\right)\right|$. Thus the $M$-particle correlation is rewritten as

$$
g_{M}\left(x_{1}, \ldots, x_{M}\right)=\frac{N !}{(N-M) !} \frac{\int_{0}^{L} \ldots \int_{0}^{L} d x_{M+1}^{F} \ldots d x_{N}^{F}\left|\psi_{N}^{F}\right|^{2}}{\int_{0}^{L} \ldots \int_{0}^{L} d x_{1}^{F} \ldots d x_{N}^{F}\left|\psi_{N}^{F}\right|^{2}},
$$

where $N$-particle fermion wave function, $\psi_{N}^{F}$ has a determinant form and norm of the wave function can be expressed as $\left|\psi_{N}^{F}\right|^{2}=\left.\operatorname{det}\left(\frac{\sin \left(N \pi\left(x_{j}^{F}-x_{l}^{F}\right) / L\right)}{\sin \left(\pi\left(x_{j}^{F}-x_{l}^{F}\right) / L\right)}\right)\right|_{j, l=1, \ldots, N}$ and the normalization factor $\int_{0}^{L} \ldots \int_{0}^{L} d x_{1}^{F} \ldots d x_{N}^{F}\left|\psi_{N}^{F}\right|^{2}=N ! L^{N}$. By changing the variable as $t_{j}=2 \pi x_{j}^{F} / L$, Eq. (18) becomes

$$
g_{M}\left(t_{1}, \ldots, t_{M}\right)=\frac{(2 \pi)^{M}}{(N-M) ! L^{M}} \int_{0}^{2 \pi} \ldots \int_{0}^{2 \pi} d t_{M+1} \ldots d t_{N}\left|\operatorname{det}\left(\frac{\sin \left(N\left(t_{j}-t_{l}\right) / 2\right)}{2 \pi \sin \left(\left(t_{j}-t_{l}\right) / 2\right)}\right)\right|_{j, l=1, \ldots, N} .
$$


Theorem: [67], Let $K(x, y)$ be a function with real, complex or quaternion values, such that $K^{*}(x, y)=K(y, x)$, where $K^{*}=K$ if $K$ is real, $K^{*}$ is the complex conjugate of $K$ if it is complex, and $K^{*}$ is dual of $K$ if it is a quaternion. Assume that

$$
\int K(x, y) K(y, z) d y=K(x, z)+\lambda K(x, z)-K(x, z) \lambda
$$

or symbolically

$$
K * K=K+\lambda K-K \lambda
$$

with $\lambda$ a constant quaternion. Let $\left[K\left(x_{i}, x_{j}\right)\right]_{N}$ denote the $N \times N$ matrix with its $(i, j)$ element equal to $K\left(x_{i}, x_{j}\right)$. Then

$$
\int \operatorname{det}\left[K\left(x_{i}, x_{j}\right)\right]_{N} d x_{N}=(A-N+1) \operatorname{det}\left[K\left(x_{i}, x_{j}\right)\right]_{N-1}
$$

where $A=\int K(x, x) d x$. When $K(x, y)$ real or complex the variable $\lambda$ vanishes.

We further observe that the elements of the determinant in the Eq. (19) is satisfied

$$
\begin{aligned}
\int_{0}^{2 \pi} \frac{\sin \left(N\left(t_{j}-t_{j}\right) / 2\right)}{2 \pi \sin \left(\left(t_{j}-t_{j}\right) / 2\right)} d t_{j} & =N \\
\int_{0}^{2 \pi} \frac{\sin \left(N\left(t_{j}-t_{l}\right) / 2\right)}{2 \pi \sin \left(\left(t_{j}-t_{l}\right) / 2\right)} \cdot \frac{\sin \left(N\left(t_{l}-t_{n}\right) / 2\right)}{2 \pi \sin \left(\left(t_{l}-t_{n}\right) / 2\right)} d t_{l} & =\frac{\sin \left(N\left(t_{j}-t_{n}\right) / 2\right)}{2 \pi \sin \left(\left(t_{j}-t_{n}\right) / 2\right)}
\end{aligned}
$$

and $A=N$ is a constant. Therefore, according to the theorem, the $M$-partical non-local correlation function can be written as

$$
\begin{aligned}
g_{M}\left(t_{1}, \ldots, t_{M}\right) & =\frac{(2 \pi)^{M}}{L^{M}}\left|\operatorname{det}\left(\frac{\sin \left(N\left(t_{j}-t_{l}\right) / 2\right)}{2 \pi \sin \left(\left(t_{j}-t_{l}\right) / 2\right)}\right)\right|_{j, l=1, \ldots, M}, \\
g_{M}\left(x_{1}, \ldots, x_{M}\right) & =\left|\operatorname{det}\left(\frac{\sin \left(N \pi\left(x_{j}-x_{l}\right) / L\right)}{L \sin \left(\pi\left(x_{j}-x_{l}\right) / L\right)}\right)\right|_{j, l=1, \ldots, M},
\end{aligned}
$$

which can be used for calculating different nonlocal correlation functions of Tonks-Girardeau Bose gas.

\section{III.2 Subleading contribution in 2-body correlation $g_{2}$}

The Forrester et.al. 30] have derived the $O(1 / c)$ oder of correction to the two-body non-local correlation function by expanding the momentum of bosons in $1 D$ Lieb-Liniger model with a finitely strong repulsion. This subleading contribution can also be retrieved by using the fractional statistics following their analytical process. When we consider the non-local correlations, the positions of particles (i.e. the permutation orders in the wave function) are very important. In the local correlation function, we used the GES, $k_{i}=\alpha k_{i}^{F}$, the coordinates of the particles are demanded by a rescaling $x_{i} \sim x_{i}^{F} / \alpha$. In contrast, such a rescaling of the particles' coordinates in a calculation of the non-local correlation function works only for a strong repulsion limit, i.e. $x_{i} / c \ll 1$. For calculating the non-local correlation function, we will use Forrester et.al. [30] expansion method in term of the statistical parameter $\alpha$.

For our convenience, we fix the number of total particles $N+2$ in the domain $0 \leq y \leq x_{1} \leq \ldots \leq x_{j} \leq x \leq x_{j+1} \leq$ $\ldots \leq x_{N} \leq L$ and label the particle positions as $\left(y, x_{1}, \ldots, x_{j}, x, x_{j+1}, \ldots, x_{N}\right)=\left(\tilde{x}_{1}, \tilde{x}_{2}, \ldots, \tilde{x}_{N+2}\right)$. Then, two-body non-local correlation function reads

$$
g_{2}^{N+2}(y, x)=\frac{(N+2) !}{N !} \frac{\int_{0}^{L} \cdots \int_{0}^{L} d x_{1}, \ldots, d x_{N}\left|\psi_{N+2}\right|^{2}}{\int_{0}^{L} \cdots \int_{0}^{L} d y d x d x_{1}, \ldots, d x_{N}\left|\psi_{N+2}\right|^{2}} .
$$

For our convenience in notation, we can denote the wave function in Eq. (5) as

$$
\begin{aligned}
\psi_{N+2} & \equiv \psi\left(y, x_{1}, \ldots, x_{j}, x, x_{j+1}, \ldots, x_{N}\right)=\psi\left(\tilde{x}_{1}^{F}, \ldots, \tilde{x}_{N+2}^{F}\right) \\
& =\left(1+(\alpha-1) \sum_{l=1}^{N+2} \tilde{x}_{l}^{F} \frac{\partial}{\partial \tilde{x}_{l}^{F}}+\frac{1}{c} \sum_{l=1}^{N+2}(2 l-(N+2)-1) \frac{\partial}{\partial \tilde{x}_{l}^{F}}\right) \psi_{N+2}^{F} .
\end{aligned}
$$


Thus, the operators in the wave function can be expanded as

$$
\begin{aligned}
\sum_{l=1}^{N+2} \tilde{x}_{l}^{F} \frac{\partial}{\partial \tilde{x}_{l}^{F}}= & y \frac{\partial}{\partial y}+x \frac{\partial}{\partial x}+\sum_{l=1}^{N} x_{l} \frac{\partial}{\partial x_{l}} \\
\sum_{l=1}^{N+2}(2 l-(N+2)-1) \frac{\partial}{\partial \tilde{x}_{l}^{F}}= & -(N+1) \frac{\partial}{\partial y}+\sum_{l=1}^{j}(2 l-N-1) \frac{\partial}{\partial x_{l}}+(2(j+1)-N-1) \frac{\partial}{\partial x} \\
& +\sum_{l=j+1}^{N}(2(l+1)-N-1) \frac{\partial}{\partial x_{l}} .
\end{aligned}
$$

Let $y=0$, and $\psi_{N+2}^{F}$ depends only on $x_{1}, \ldots, x_{N}$ variables. At the ground state, the system has zero total momentum, i.e. $\frac{\partial}{\partial y}=-\left(\frac{\partial}{\partial x}+\sum_{l=1}^{N} \frac{\partial}{\partial x_{l}}\right)$. The wave function of free fermions vanishes when two particles coincide, i.e. $\psi_{N+2}^{F}=0$ at $x_{l}=0, L, x_{j}(j \neq l)$. Therefore,

$$
\begin{aligned}
\int_{\tilde{x}_{1} \leq \ldots \leq \tilde{x}_{j} \ldots \leq \tilde{x}_{N+2}} d x_{l} \frac{\partial}{\partial x_{l}}\left|\psi_{N+2}^{F}\right|^{2} & =0, \quad \text { and } \\
\int_{\tilde{x}_{1} \leq \ldots \leq \tilde{x}_{j} \ldots \leq \tilde{x}_{N+2}} d x_{l} x_{l} \frac{\partial}{\partial x_{l}}\left|\psi_{N+2}^{F}\right|^{2} & =-\int_{\tilde{x}_{1} \leq \ldots \leq \tilde{x}_{j} \ldots \leq \tilde{x}_{N+2}}\left|\psi_{N+2}^{F}\right|^{2} d x_{l} .
\end{aligned}
$$

Taking together these conditions with the Eq. (29), we may show that $\mathcal{N}^{2}=\alpha^{-(N+1)}(N+1) ! L^{N+1}$ and

$g_{2}^{N+2}(0, x)=\alpha^{(N+1)}\left[1+(\alpha-1)\left(x \frac{\partial}{\partial x}-N\right)\right] g_{2}^{N+2, F}(0, x)+\frac{1}{\mathcal{N}^{2}} \frac{2}{c} \int \ldots \int_{\tilde{x}_{1} \leq \ldots \leq \tilde{x}_{N+2}} d x_{1}, \ldots, d x_{N}(j+1) \frac{\partial}{\partial x} \psi_{N+2}^{F}(0, x)$,

where $\psi_{N+2}^{F}\left(0, x_{1}, \ldots, x, \ldots, x_{N}\right) \equiv \psi_{N+2}^{F}(0, x)$.

Furthermore, the coefficient of $g_{2}^{N+2, F}(0, x)$ in Eq. (32) can be simplified in term of the GES parameter $\alpha$. Eq. (32) has been derived in terms of $1 / c$ corrections [30]. A detailed calculation of the integral term (in Appendix C), gives

$$
\frac{1}{\mathcal{N}^{2}} \int \ldots \int_{\tilde{x}_{1} \leq \ldots \leq \tilde{x}_{N+2}}(j+1) d x_{1}, \ldots, d x_{N} \frac{\partial}{\partial x} \psi_{N+2}^{F}(0, x)=\alpha^{N+1}\left(\frac{\partial}{\partial x} g_{2}^{N+2, F}(0, x)+\frac{\partial}{\partial x} \int_{0}^{x} d x_{l} g_{3}^{N+2, F}\left(0, x, x_{l}\right)\right),
$$

where $g_{2}^{N+2, F}(0, x)$ and $g_{3}^{N+2, F}\left(0, x, x_{l}\right)$ are the two-body and three-body non-local correlation functions of $N+1$ free fermions, which have been given in Eq. (25). Finally, the Eq. (32) can be viewed as the two-body non-local correlation function of $N$ particles, namely

$$
g_{2}^{N}(0, x)=\alpha g_{2}^{N, F}(0, x)+(\alpha-1) x \frac{\partial}{\partial x} g_{2}^{N, F}(0, x)+\frac{2}{c} \frac{\partial}{\partial x} g_{2}^{N, F}(0, x)+\frac{2}{c} \frac{\partial}{\partial x} \int_{0}^{x} d x_{l} g_{3}^{N, F}\left(0, x, x_{l}\right) .
$$

We observe that the two-body correlation function essentially depends on the two-body and higher order non-local correlation functions of the free fermions and their derivatives, which provide insights into the many-body correlations in strongly interacting bosons.

\section{LARGE MOMENTUM TAIL OF THE MOMENTUM DISTRIBUTION AND TAN'S CONTACT RELATIONS}

Various methods can be used to calculate the contact [39 41, 43 45], for example, it can be obtained from the free energy or ground state energy via the Helmann-Feynmann theorem [46], or from the single particle correlation function [47], from the operator product expansion field theory method [48], etc.

\section{IV.1 Large momentum Tail of the momentum distribution}

The single-particle momentum distribution, $w(p)$ at momentum $p=\hbar k$, is the Fourier transform of the one-particle density matrix $\rho\left(x_{j}, x_{k}\right)$. In order to build up a connection between the Tan's contact and the power law decay with 
distance in large momentum tail of momentum distribution, we first calculate the wave function in momentum space. To this end, we introduce the centre of mass coordinate, $R=\left(x_{j}+x_{k}\right) / 2$ and relative coordinate, $x_{k j}=x_{k}-x_{j}$, of the $j, k$ pair of particles with other fixed $N-2$ coordinates $\left\{x_{i}\right\}_{i \neq j, k}$. The $k^{t h}$ particle in the neighbor of $j^{\text {th }}$ particle, such as $x_{1} \leq x_{2} \leq x_{3} \leq \ldots \leq x_{k-1} \leq x_{j} \leq x_{k} \leq x_{k+1} \leq \ldots \leq x_{N}$ and $x_{1} \leq x_{2} \leq x_{3} \leq \ldots \leq x_{k-1} \leq x_{k} \leq x_{j} \leq$ $x_{k+1} \leq \ldots \leq x_{N}$, the wave function for $1 \mathrm{D}$ strongly interacting bosons in Eq. (3) can be approximately written as

$$
\psi^{B}\left(x_{1}, \ldots, x_{N}\right)=\frac{1}{\mathcal{N}} \mathcal{A}(c) \sum_{p}(-1)^{p} e^{\mathrm{i}\left(k_{p_{k}}+k_{p_{j}}\right) R}\left(1+\frac{\mathrm{i}}{2}\left(k_{p_{k}}-k_{p_{j}}\right) x_{k j}-\frac{1}{8}\left(k_{p_{k}}-k_{p_{j}}\right)^{2} x_{k j}^{2}+O\left(x_{k j}^{3}\right)\right) e^{\mathrm{i} i \neq k, j ; i=1} \sum_{i p_{i} x_{i}}^{N} .
$$

Following symmetric conditions, the Eq. (35) can be represented in general forms as

$$
\left.\psi^{B}\left(x_{1}, \ldots, x_{N}\right)\right|_{x_{k}=x_{j}^{+}} \approx \frac{1}{\mathcal{N}}\left(1+\frac{1}{2}\left(\frac{\partial}{\partial x_{k}}-\frac{\partial}{\partial x_{j}}\right)\left|x_{k j}\right| \operatorname{sgn}\left(x_{k j}\right)+\frac{1}{8}\left(\frac{\partial}{\partial x_{k}}-\frac{\partial}{\partial x_{j}}\right)^{2} x_{k j}^{2}\right) \psi,
$$

for bosons in the $1 D$ Lieb-Liniger model, where $\operatorname{sgn}(x)$ is +1 for $x>0$ and -1 for $x<0, \psi$ that corresponds to the wave function Eq. (3) at $x_{k}=x_{j}^{+}$.

The Fourier transform of the one-particle correlation function is the momentum distribution:

$$
w(p)=\int d x d x^{\prime} g_{1}\left(x ; x^{\prime}\right) e^{-\mathrm{i} p\left(x-x^{\prime}\right)},
$$

where $p=(2 \pi / L) s$ and $s$ is an integer. This is equivalent to calculating first the many-particle wave function in momentum space for one atom and then integrating out the rest of the coordinates from its square modulus. In order to calculate the asymptotic behaviour (the large $p$ tail) of the momentum distribution [42], we first consider the normalized wave function with respect to the first particle in momentum space

$$
\psi^{B}\left(p, x_{2}, \ldots, x_{k}, \ldots, x_{N}\right)=\sum_{k=2}^{N} \frac{1}{\sqrt{L}} \int_{0}^{L} d x_{1} e^{-i p x_{1} / \hbar} \psi^{B}\left(x_{1}, x_{2}, \ldots, x_{k}, \ldots, x_{N}\right),
$$

where $p=(2 \pi \hbar / L) l$ where $l$ is an integer. For a repulsive interaction, the diagonal terms becomes much larger than the off-diagonal terms due to the periodic boundary condition and symmetry. For a periodic function $F_{1}\left(x_{1}, \ldots, x_{N}\right)\left(x_{k}-\right.$ $\left.x_{1}\right) \operatorname{sgn}\left(x_{k}-x_{1}\right)$, defined on the interval $[0, L]$, where $F_{1}\left(x_{1}, \ldots, x_{N}\right)=\left(\frac{\partial}{\partial x_{k}}-\frac{\partial}{\partial x_{1}}\right) \sum_{P}(-1)^{P} \exp \left(\mathrm{i} \sum_{j=1}^{N} k_{P_{j}} x_{j}\right)$ is a regular function, we thus rewrite the square modulus as

$$
\left.\left|\psi^{B}\left(p, x_{2}, \ldots, x_{k}, \ldots, x_{N}\right)\right|^{2} \approx \frac{(N-1)}{\mathcal{N}^{2} L(p / \hbar)^{4}}\left(1+\frac{1}{c} \sum_{j=1}^{N}(2 j-N-1) \frac{\partial}{\partial x_{j}}\right)\left|F_{1}\left(x_{1}, \ldots, x_{N}\right)\right|^{2}\right|_{x_{k}=x_{1}}+O\left(\frac{\hbar^{5}}{p^{5}}\right) .
$$

After a length calculation, the asymptotic behaviour of the momentum distribution is given by

$$
\begin{aligned}
w(p) \stackrel{p \rightarrow \infty}{=} & N \int_{0}^{L} \ldots \int_{0}^{L} d x_{2} \ldots d x_{k} \ldots d x_{N}\left|\psi^{B}\left(p, x_{2}, \ldots, x_{k}, \ldots, x_{N}\right)\right|^{2} \\
& =\left.N \int_{0}^{L} \ldots \int_{0}^{L} d x_{2} \ldots d x_{N}\left[\frac{(N-1)}{(p / \hbar)^{4} \mathcal{N}^{2} L}\left(1+\frac{1}{c} \sum_{j=1}^{N}(2 j-N-1) \frac{\partial}{\partial x_{j}}\right)\left|F_{1}\left(x_{1}, \ldots, x_{N}\right)\right|^{2}\right]\right|_{x_{k}=x_{1}^{+}} \\
& =\left.\frac{N(N-1)}{(p / \hbar)^{4} \mathcal{N}^{2} L} \int_{0}^{L} \ldots \int_{0}^{L} d x_{2} \ldots d x_{N}\left[\left(1+\frac{1}{c} \sum_{j=1}^{N}(2 j-N-1) \frac{\partial}{\partial x_{j}}\right)\left|F_{1}\left(x_{1}, \ldots, x_{N}\right)\right|^{2}+O\left(\frac{1}{c^{2}}\right)\right]\right|_{x_{k}=x_{1}^{+}}
\end{aligned}
$$

Applying the GSE relation, $k_{P_{j}}=k_{P_{j}}^{F} \alpha$, making a rescaling $x_{j}=x_{j}^{F} / \alpha$, and after a lengthy calculation, we obtain the momentum distribution of $1 D$ bosons up to order $1 / c$

$\left.w(p) \stackrel{p \rightarrow \infty}{\approx} \frac{\alpha^{2}}{L(p / \hbar)^{4}} \int_{0}^{\alpha L} d x_{k}^{F}\left(1+\frac{\alpha}{c} \sum_{j=1}^{N}(2 j-N-1) \frac{\partial}{\partial x_{j}^{F}}\right)\left(\frac{\partial}{\partial x_{k}^{F}}-\frac{\partial}{\partial x_{1}^{F}}\right)\left(\frac{\partial}{\partial y_{k}^{F}}-\frac{\partial}{\partial y_{1}^{F}}\right) g_{2}^{F}\left(x_{1}^{F}, x_{k}^{F} ; y_{1}^{F}, y_{k}^{F}\right)\right|_{x_{k}^{F} \rightarrow x_{1}^{F}+} ^{(42)}$

where $g_{2}^{F}$ is fermions two-particle correlation, Up to order $1 / c$, the normalization factor for strongly interacting $1 D$ bosons has been calculated in [19] as $\mathcal{N}^{2}=\left.\alpha^{1-N} \int_{0}^{L} \ldots \int_{0}^{L} d x_{1}^{F} \ldots d x_{N}^{F}\left|\sum_{P}(-1)^{P} \exp \left(\mathrm{i} \sum_{j=1}^{N} k_{P_{j}}^{F} x_{j}^{F}\right)\right|^{2}\right|_{x_{k}^{F}=x_{1}^{F}}$. 
By applying Wicks theorem and getting partial derivatives, the asymptotic behaviuor of the momentum distribution becomes,

$$
w(p) \stackrel{p \rightarrow \infty}{=} \frac{g_{2}(0,0) \gamma^{2} n^{2}}{(p / \hbar)^{4}}=\frac{4 \alpha^{3} \pi^{2} n^{4}}{3(p / \hbar)^{4}}+O\left(\frac{1}{c^{2}}, \frac{1}{(p / \hbar)^{5}}\right) .
$$

In the above calculation Eq. (42), we have proved that the leading order part gives the universal asymptotic behaviour Eq. (43), whereas the second term in Eq. (42) is zero. According to the definition of momentum distribution, the Eq. (43) is equal to the relation given in [36] and [37]. Moreover, the Hellmannn-Feynman theorem relates to the derivative of the energy with respect to the interacting strength. So we have a two-particle local correlation function $g_{2}^{B}\left(x_{1}, x_{1}\right)=n^{2} e^{\prime}(\gamma)$

$$
w(p) \stackrel{p \rightarrow \infty}{=} \frac{e^{\prime}(\gamma) n^{4} \gamma^{2}}{(p / \hbar)^{4}}
$$

where the derivative of total energy, $e^{\prime}(\gamma) \approx \frac{\hbar^{2}}{2 m} \frac{\pi^{2} \alpha^{2}}{3}[66$. The result of Eq. (43) shows that the tail of the momentum distribution decays with a power of $1 /(p / \hbar)^{4}$ and it has a sub-leading contribution determined by the GES parameter $\alpha$.

\section{IV.2 THE CONTACT RELATION}

A powerful universal relation connects the strength of short-range two-particle correlations to the thermodynamics of a many-particle system with zero-range interaction [55]. The contact allows for a simple analytic expression that unveils how to scale the momentum distribution function at large momentum [54]. We recall the Lemma discussed in [36, 46] and [68].

Lemma 1 Consider a function absolutely integrable, vanishing at infinity which has a singularity of the type $f(x)=\left|x-x_{0}\right|^{\alpha} F(x)$ at $x_{0}$ with $F(x)$ analytical and $\alpha>-1, \alpha \neq, 0,2,4, \ldots$. Then we have

$$
\lim _{k \rightarrow \infty} \int_{-\infty}^{\infty} e^{-\mathrm{i} k x} f(x) d x \approx 2 \cos \frac{\pi}{2}(\alpha+1) \tau(\alpha+1) \frac{e^{-\mathrm{i} k x_{0}}}{|k|^{\alpha+1}} F\left(x_{0}\right)+O\left(1 /|k|^{\alpha+2}\right) .
$$

In the case of multiple singular points of the same type, the asymptotic behaviour of the integral is given by the sum of all the corresponding contributions given by the right hand side of the Eq. (45).

Considering discontinuity of the derivative wave function, the Eq. (36) is given by

$$
\psi^{B}\left(x_{1}, \ldots, x_{N}\right)=\frac{1}{\mathcal{N}}\left(1-\frac{\left|x_{k j}\right|}{a_{1 D}} \operatorname{sgn}\left(x_{k j}\right)+\frac{2 x_{k j}^{2}}{a_{1 D}^{2}}+O\left(x_{k j}\right)^{3}\right) \psi,
$$

where $c=-2 / a_{1 D}$. Applying the Lemma 1 to the Eq. (46) and after changing integration variables as $d x_{1} \sim d x$ and $d x_{2} \sim d R$ at $x_{1} \rightarrow x_{k}$, we find that the function $\psi$ is a function of $R$ and $X$, where the set of fixed coordinates $X=\left\{x_{i}\right\}_{i=2 \ldots, k-1, k+1, \ldots, N}$. Thus we may calculate the expression Eq. (38) as

$$
\begin{aligned}
\left|\psi^{B}\left(p, x_{2}, \ldots, x_{k}, \ldots, x_{N}\right)\right|^{2} & \approx \frac{(N-1)}{\mathcal{N}^{2} L}\left[\int_{0}^{L} d x e^{-\mathrm{i} p x / \hbar}\left(1-\frac{|x|}{a_{1 D}}\right)|\psi(R, R, X)|\right]^{2} \\
& \stackrel{p \rightarrow \infty}{=} \frac{(N-1)}{\mathcal{N}^{2} L}\left(\frac{4}{a_{1 D}^{2}(p / \hbar)^{4}}\right)|\psi(R, R, X)|^{2} .
\end{aligned}
$$

In the above equation, we have neglected the off-diagonal terms which vanish faster than the diagonal terms. From Eq. (40), the large momentum tail of the momentum distribution is obtained as

$$
w(p) \stackrel{p \rightarrow \infty}{=} \frac{4}{a_{1 D}^{2} L(p / \hbar)^{4}} C_{2},
$$

where the two-particle contact defined by $C_{2}=\int_{0}^{L} d R g_{2}(R, R)$. Here the two-particle contact is given by $C_{2}=$ $4 \pi^{2} n N \gamma^{-2} \alpha^{3} / 3$. Whereas the two-particle local correlation function reads $g_{2}(0,0)=4 \pi^{2} n^{2} \alpha^{3} /\left(3 \gamma^{2}\right)$ for a finitely 
strong repulsive Bose gas at zero temperature, further confirming Eq. (11). This result agrees with the result which has been derived by Yuta et.al. in [52]. Moreover, in a weakly coupling regime, the two-particle local correlation functions is given by $g_{2}(0,0)=n^{2}(1-2 \sqrt{\gamma} / \pi)$. So the two-particle contact can be obtained as $C_{2}=n N(1-2 \sqrt{\gamma} / \pi)$ for the $1 \mathrm{D}$ weakly interacting bosons.

\section{IV.3 STATIC STRUCTURE FACTOR}

The structure factor is a property that defines how an ensemble of atoms scatters incident radiation. Experimentally, it is usually measured by two-photon Bragg scattering. [42]. Due to the translational invariance of the system, the density is constant [52], the static structure factor is defined as

$$
S(k)=1+\frac{1}{N} \int d x_{k} \int d x_{j} e^{-\mathrm{i} k\left(x_{k}-x_{j}\right)}\left(g_{2}\left(x_{k}, x_{j}\right)-n\left(x_{k}\right) n\left(x_{j}\right)\right),
$$

where $n\left(x_{i}\right)$ is the density. Changing integration variables as before, we have

$$
\begin{aligned}
S(k) & =1+\frac{1}{N} \int_{0}^{L} d x \int_{0}^{L} d R e^{-\mathrm{i} k x}\left(\left(1-\frac{2|x|}{a_{1 D}}\right) g_{2}\left(R, R^{+}\right)-n^{2}\right) \\
& =1+\frac{4}{N a_{1 D} k^{2}} C_{2},
\end{aligned}
$$

where $C_{2}=\int_{0}^{L} d R g_{2}\left(R, R^{+}\right)$, also see [52]. By using Eq. (25), the two-particle non-local correlation function of the $1 \mathrm{D}$ Bose gas in strongly interacting regime, i.e. $c \rightarrow \infty$, is given by

$$
g_{2}\left(R, R^{+}\right) \approx n^{2}\left[1-\left(\frac{\sin \left(k^{F} x\right)}{k^{F} x}\right)^{2}\right]
$$

Here, $x$ and $k^{F}=\pi n$ are the relative coordinate and the cut-off Fermi momentum. Thus the structure factor can be determined by Eq. (50), namely

$$
S(k)= \begin{cases}1 ; & \text { for }|k|>2 k^{F} \\ \frac{|k|}{2 k^{F}} ; & \text { for }|k|<2 k^{F}\end{cases}
$$

In fact, in the low-momentum and the infinitely repulsive regime, the static structure factor has a linear dependence of $k$. In the TG regime this behaviour converts to $S(k)=|k| / 2 k^{F}$. For high momentum, the structure factor always converges to 1 .

\section{CONCLUSION}

In summary, we have presented a pedagogical study of various higher order local and non-local correlation functions of $1 \mathrm{D}$ Lieb-Liniger Bose gas. Using the Bethe wave function, we have studied the non-local $2 M$ point correlation functions and large momentum tail of momentum distributions in term of the fractional statistical parameter $\alpha$ for the system with a strong repulsion. We have also discussed the higher order correlation functions for the weakly interacting Bose gas via the Bethe ansatz wave function. It turns out that the leading order of the large-momentum tail is determined by the contact, which is related to the short-distance behaviour of the two-body density matrix. The two-body density matrix has been given in term of the fractional statistical parameter $\alpha$, giving the subleading order $1 / c$ correction in the strong coupling regime, where the interaction strength $c \gg 1$. Our results show that the fractional statistical parameter $\alpha$ attributes to the sub-leading contributions of the local, non-local correlation functions, two-body contact and static structure factor, etc. Our method is applicable to other exactly solvable models.

Acknowledgments: We thank Xiaoguo Yin, Yu-Zhu Jiang, Shina Tan for helpful discussion. This work is supported by the NSFC under grant numbers 11374331, the key NSFC grant No. 11534014 and the National Key R\&D Program of China No. 2017YFA0304500. This work has been partially supported by CAS-TWAS President's Fellowship for International PhD students. 


\section{APPENDIX A}

The Betha ansatz wave function given in Eq. (2)

$$
\psi\left(x_{1}, \ldots, x_{N}\right)=\sum_{P}(-1)^{P}\left[\prod_{1 \leq i<j \leq N}\left(\frac{\mathrm{i} c+k_{P_{i}}-k_{P_{j}}}{\mathrm{i} c-k_{P_{i}}+k_{P_{j}}}\right)^{1 / 2}\right] e^{\mathrm{i} \sum_{j=1}^{N} k_{P_{j}} x_{j}} .
$$

Using the Bethe ansatz equation, we have

$$
\begin{aligned}
(-1)^{P} A(P) \equiv(-1)^{P} \prod_{i<j}\left(\frac{\mathrm{i} c+k_{P_{i}}-k_{P_{j}}}{\mathrm{i} c-k_{P_{i}}+k_{P_{j}}}\right)^{1 / 2} & =(-1)^{P} \prod_{i<j}\left[-2 \operatorname{Tan}^{-1}\left(\frac{k_{p_{i}}-k_{p_{j}}}{c}\right)\right]^{1 / 2} \\
& \equiv(-1)^{P} \prod_{i<j}\left(e^{\mathrm{i} \theta\left(k_{p_{i}}-k_{p_{j}}\right)}\right)^{1 / 2} \\
& =e^{\mathrm{i} \frac{\pi}{2}\left(1-(-1)^{P}\right)} e^{\frac{\mathrm{i}}{2} \sum_{i<j} \theta\left(k_{p_{i}}-k_{p_{j}}\right)} \\
& =\exp \left(\mathrm{i} \frac{\pi}{2}\left(1-(-1)^{P}\right)+\frac{\mathrm{i}}{2} \sum_{i<j} \theta\left(k_{p_{i}}-k_{p_{j}}\right)\right) .
\end{aligned}
$$

Furthermore we consider the following relation

$$
\operatorname{Arctan}(x)+\operatorname{Arctan}(1 / x)=\left\{\begin{array}{l}
\pi / 2 \text { for } x>0 \\
-\pi / 2 \text { for } x<0
\end{array}\right.
$$

Then, we have

$$
\theta\left(k_{i}-k_{j}\right)=-2 \operatorname{Tan}^{-1}\left(\frac{k_{i}-k_{j}}{c}\right)=-\frac{\left(k_{i}-k_{j}\right)}{\left|k_{i}-k_{j}\right|} \pi+\frac{2 c}{k_{i}-k_{j}} .
$$

Taking $q_{j}=\sqrt{\frac{L}{2 c}} k_{j}$,

$$
\theta\left(k_{i}-k_{j}\right)=\theta\left(\sqrt{\frac{2 c}{L}}\left(q_{i}-q_{j}\right)\right)=-\frac{\left(q_{i}-q_{j}\right)}{\left|q_{i}-q_{j}\right|} \pi+\sqrt{2 c L} F\left(q_{i}-q_{j}\right),
$$

where $F\left(q_{i}-q_{j}\right)=\left(q_{i}-q_{j}\right)^{-1}$. Therefore, Eq. (55) becomes

$$
(-1)^{P} A(P)=\exp \left(\mathrm{i} \frac{\pi}{2}\left(1-(-1)^{P}\right)+\frac{\mathrm{i}}{2} \sum_{i<j}\left[-\frac{\left(q_{i}-q_{j}\right)}{\left|q_{i}-q_{j}\right|} \pi+\sqrt{2 c L} F\left(q_{i}-q_{j}\right)\right]\right) .
$$

Using Eq. (59), we consider the two permutations of the system with 3 particles.

$$
\begin{aligned}
(-1)^{P} A(123) & =\exp \left(\mathrm{i} \frac{\pi}{2}(1-1)+\frac{\mathrm{i}}{2} \sum_{i<j}\left[-\frac{\left(q_{i}-q_{j}\right)}{\left|q_{i}-q_{j}\right|} \pi+\sqrt{2 c L} F\left(q_{i}-q_{j}\right)\right]\right) \\
& =\exp \left(\frac{\mathrm{i}}{2} 3 \pi\right) \exp \left(\mathrm{i} \sqrt{\frac{c L}{2}}\left[F\left(q_{1}-q_{2}\right)+F\left(q_{1}-q_{3}\right)+F\left(q_{2}-q_{3}\right)\right]\right) . \\
(-1)^{P} A(213) & =\exp \left(\mathrm{i} \frac{\pi}{2}(1-(-1))+\frac{\mathrm{i}}{2} \sum_{i<j}\left[-\frac{\left(q_{i}-q_{j}\right)}{\left|q_{i}-q_{j}\right|} \pi+\sqrt{2 c L} F\left(q_{i}-q_{j}\right)\right]\right) \\
& =\exp \left(\frac{\mathrm{i}}{2} 3 \pi\right) \exp \left(\mathrm{i} \sqrt{\frac{c L}{2}}\left[F\left(q_{2}-q_{1}\right)+F\left(q_{2}-q_{3}\right)+F\left(q_{1}-q_{3}\right)\right]\right) .
\end{aligned}
$$

Therefore, the general form of amplitude with permutation's sign of the wave function in the weak coupling regime can be obtained as

$$
(-1)^{P} A(p)=\exp \left(\mathrm{i} \sqrt{\frac{c L}{2}} \sum_{i<j}\left[F\left(q_{i}-q_{j}\right)\right]\right)
$$


Finally, we rewrite the Bethe ansatz wave function, Eq. (3) in the weak coupling regime as

$$
\begin{aligned}
\psi\left(x_{1}, \ldots, x_{N}\right)= & \sum_{P} \exp \left(\mathrm{i} \sqrt{\frac{c L}{2}} \sum_{i<j}\left[\sqrt{2 c L} F\left(q_{P_{i}}-q_{P_{j}}\right)\right]\right) \exp \left(\mathrm{i} \sum_{j=1}^{N} \sqrt{\frac{2 c}{L}} q_{P_{j}} x_{j}\right) \\
= & \sum_{P}\left[1+\mathrm{i}\left(\sqrt{\frac{c L}{2}} \sum_{i<j} F\left(q_{P_{i}}-q_{P_{j}}\right)+\sqrt{\frac{2 c}{L}} \sum_{j=1}^{N} q_{P_{j}} x_{j}\right)\right. \\
& \left.\quad-\frac{1}{2}\left(\sqrt{\frac{c L}{2}} \sum_{i<j} F\left(q_{P_{i}}-q_{P_{j}}\right)+\sqrt{\frac{2 c}{L}} \sum_{j=1}^{N} q_{P_{j}} x_{j}\right)^{2}+\ldots\right] \\
= & N !-\frac{c}{2} Y\left(x_{1}, \ldots, x_{N}\right),
\end{aligned}
$$

where $Y\left(x_{1}, \ldots, x_{N}\right)=\sum_{P}\left(\sqrt{\frac{L}{2}} \sum_{i<j} F\left(q_{P_{i}}-q_{P_{j}}\right)+\sqrt{\frac{2}{L}} \sum_{j=1}^{N} q_{P_{j}} x_{j}\right)^{2}$, since the symmetric condition gives $\sum_{P} \sum_{i<j} F\left(q_{P_{i}}-q_{P_{j}}\right)=0$ and $\sum_{P} \sum_{j=1}^{N} q_{P_{j}}=0$

\section{APPENDIX B}

Let us represent the field operator $\hat{\Psi}$ as a sum of the condensate part $\psi_{0}$ and a non-condensate part $\psi^{\prime}$ such as

$$
\hat{\Psi}=\psi_{0}+\psi^{\prime} .
$$

In the uniform case, we may let the condensate contain a macroscopic number of particles $N_{0}$. Consider the density of the system is $n=n_{0}+n^{\prime}$ where $n_{0}=\left\langle\psi_{0} \psi_{0}^{*}\right\rangle=\left|\psi_{0}\right|^{2}$ is the condensed particles density and $n^{\prime}=\left\langle\psi^{\prime \dagger} \psi^{\prime}\right\rangle$ is the non condensed particles density. Considering the $M^{\text {th }}$ order density of the system with respect to the first order of $\psi^{\prime}$, we have

$$
\begin{aligned}
n^{M} & =\left(\left|\psi_{0}\right|^{2}\right)^{M}+M\left(\left|\psi_{0}\right|^{2}\right)^{M-1}\left\langle\psi^{\prime \dagger} \psi^{\prime}\right\rangle+O\left(\left\langle\psi^{\prime \dagger} \psi^{\prime}\right\rangle\right)^{2}, \\
\Rightarrow\left(\left|\psi_{0}\right|^{2}\right)^{M} & =n^{M}-M\left(\left|\psi_{0}\right|^{2}\right)^{M-1}\left\langle\psi^{\prime \dagger} \psi^{\prime}\right\rangle \\
& =n^{M}-M n^{M-1}\left\langle\psi^{\prime \dagger} \psi^{\prime}\right\rangle+O\left(\left\langle\psi^{\prime \dagger} \psi^{\prime}\right\rangle\right)^{2} .
\end{aligned}
$$

The $M$ particle correlation function for $1 D$ bosons at weak coupling limit reads

$$
g_{M}^{w}=\left\langle\left(\psi_{0}^{*}+\psi^{\prime \dagger}\right)^{M}\left(\psi_{0}+\psi^{\prime}\right)^{M}\right\rangle .
$$

Expanding the $M^{t h}$ order in Eq. (67) and substituting Eq. (66) into the above equation, we obtain

$$
\begin{aligned}
g_{M}^{w}= & \left\langle\left(\psi_{0}^{* M}+M \psi_{0}^{*(M-1)} \psi^{\prime \dagger}+\frac{1}{2} M(M-1) \psi_{0}^{*(M-2)} \psi^{\prime \dagger 2}+O\left(\psi^{\prime \dagger 3}\right)\right)\right. \\
& \left.\times\left(\psi_{0}^{M}+M \psi_{0}^{M-1} \psi^{\prime}+\frac{1}{2} M(M-1) \psi_{0}^{(M-2)} \psi^{\prime 2}+O\left(\psi^{\prime 3}\right)\right)\right\rangle \\
= & \left\langle\left|\psi_{0}\right|^{2 M}+M^{2}\left|\psi_{0}\right|^{2(M-1)}\left(\psi^{\prime \dagger} \psi^{\prime}\right)+M(M-1)\left|\psi_{0}\right|^{2(M-1)} \psi^{\prime 2}\right\rangle \\
= & n^{M}\left(1+\frac{M(M-1)}{n}\left(\left\langle\psi^{\prime \dagger} \psi^{\prime}\right\rangle+\left\langle\psi^{\prime} \psi^{\prime}\right\rangle\right)\right) .
\end{aligned}
$$

Eq. (68) shows that $g_{M}^{w}$ depends on the normal density of non-condensed particles, $\left\langle\psi^{\prime \dagger} \psi^{\prime}\right\rangle$, and anomalous density of non-condensed particles $\left\langle\psi^{\prime} \psi^{\prime}\right\rangle$. Consider the form of non-condensate part, $\psi^{\prime}$, as

$$
\psi^{\prime}=\sum_{\nu}\left(u_{\nu} \hat{b}_{\nu} e^{-\mathrm{i} \epsilon_{\nu} t / \hbar}-v_{\nu}^{*} \hat{b}_{\nu}^{\dagger} e^{\mathrm{i} \epsilon_{\nu} t / \hbar}\right)
$$

which is known as Bogoliubov transformation. Here the index $\nu$ labels quantum states of elementary excitations, $\hat{b}_{\nu}, \hat{b}_{\nu}^{\dagger}$ are operators of excitations, $\epsilon_{\nu}$ are their eigenenergies, and $u_{\nu}, v_{\nu}$ their eigen functions. The operators $\hat{b}_{\nu}, \hat{b}_{\nu}^{\dagger}$ obey the usual boson commutation relations

$$
\hat{b}_{\nu} \hat{b}_{\nu^{\prime}}^{\dagger}-\hat{b}_{\nu^{\prime}}^{\dagger} \hat{b}_{\nu}=\delta_{\nu \nu^{\prime}}, \quad \hat{b}_{\nu} \hat{b}_{\nu^{\prime}}-\hat{b}_{\nu^{\prime}} \hat{b}_{\nu}=0 .
$$


The functions $u_{\nu}, v_{\nu}$ are normalized by the condition [13]

$$
\int\left(u_{\nu} u_{\nu}^{*}-v_{\nu} v_{\nu}^{*}\right) d x=\delta_{\nu \nu^{\prime}}
$$

Taking into account that the index $\nu$ is now the excitation wave vector $k$, we write the excitation wave function in the form $u_{\nu}=\frac{u_{k}}{\sqrt{L}} e^{\mathrm{i} k x}$ and $v_{\nu}=\frac{v_{k}}{\sqrt{L}} e^{\mathrm{i} k x}$. Consequently, we have

$$
\psi^{\prime}=\frac{1}{\sqrt{L}} \sum_{k}\left(u_{k} \hat{b}_{k} e^{\mathrm{i}\left(k x-\epsilon_{k} t / \hbar\right)}-v_{k}^{*} \hat{b}_{k}^{\dagger} e^{-\mathrm{i}\left(k x-\epsilon_{k} t / \hbar\right)}\right) .
$$

The normal and anomalous density of the non-condensate part can be calculated by using Eq. (72) at $k=k^{\prime}$ as

$$
\begin{aligned}
\left\langle\psi^{\prime \dagger} \psi^{\prime}\right\rangle & =\frac{1}{L} \sum_{k}\left[u_{k}^{2} N_{k}+v_{k}^{2}\left(1+N_{k}\right)\right], \\
\left\langle\psi^{\prime} \psi^{\prime}\right\rangle & =\frac{1}{L} \sum_{k}\left[-u_{k} v_{k}^{*}\left(1+N_{k}\right)-v_{k}^{*} u_{k} N_{k}\right],
\end{aligned}
$$

where $N_{k}$ is the occupation number for the excitations with $N_{k} \rightarrow 0$ as $T \rightarrow 0$ and commutation relations of the excitation operators are $\left\langle\hat{b}_{k^{\prime}}^{\dagger} \hat{b}_{k}\right\rangle=\delta_{k^{\prime} k} N_{k}, \quad\left\langle\hat{b}_{k^{\prime}} \hat{b}_{k}^{\dagger}\right\rangle=\delta_{k^{\prime} k}\left(1+N_{k}\right), \quad\left\langle\hat{b}_{k^{\prime}} \hat{b}_{k}\right\rangle=\left\langle\hat{b}_{k^{\prime}}^{\dagger} \hat{b}_{k}^{\dagger}\right\rangle=0$. Summation of Eq. (73) and Eq. (74)

$$
\left\langle\psi^{\prime \dagger} \psi^{\prime}\right\rangle+\left\langle\psi^{\prime} \psi^{\prime}\right\rangle=\frac{1}{L} \sum_{k}\left[u_{k}^{2} N_{k}+v_{k}^{2}\left(1+N_{k}\right)-u_{k} v_{k}\left(1+2 N_{k}\right)\right],
$$

since $v_{k}^{*}=v_{k}$. By considering up to the linear order of $\psi^{\prime}$ of field operator Eq. (64), the Gross-Pitaevskii equation can be obtain as

$$
\mathrm{i} \hbar \frac{\partial \psi^{\prime}}{\partial t}=\left(-\frac{\hbar^{2}}{2 m} \frac{\partial^{2}}{\partial x^{2}}+2 g\left|\psi_{0}\right|^{2}-\mu\right) \psi^{\prime}+g \psi_{0}^{2} \psi^{\prime \dagger}
$$

The non-condensate density yields from the eigenfunctions $u_{k}$ and $v_{k}$. In order to find $u_{k}$ and $v_{k}$, evaluate the commutator of both sides of Eq. (76) with $\hat{b}_{k}$ and repeating procedure with $\hat{b}_{k}^{\dagger}$, one can get

$$
\begin{aligned}
-\epsilon_{k} v_{k} & =\left(\frac{\hbar^{2} k^{2}}{2 m}+2 g\left|\psi_{0}\right|^{2}-\mu\right) v_{k}-g \psi_{0}^{2} u_{k}, \\
\epsilon_{k} u_{k} & =\left(\frac{\hbar^{2}}{2 m} k^{2}+2 g\left|\psi_{0}\right|^{2}-\mu\right) u_{k}-g \psi_{0}^{2} v_{k} .
\end{aligned}
$$

Here, $v^{*}=v$ and $u^{*}=u$. Substituting $\mu=n_{0} g$, and $E_{k}=\frac{\hbar^{2} k^{2}}{2 m}$, to the Eq. (177) and Eq. (178) which are called Bogoliubov-de Gennes equations for elementary excitations, are transformed to

$$
\begin{aligned}
-\epsilon_{k} v_{k} & =\left(E_{k}+g n_{0}\right) v_{k}-g n_{0} u_{k}, \\
\epsilon_{k} u_{k} & =\left(E_{k}+g n_{0}\right) u_{k}-g n_{0} v_{k} .
\end{aligned}
$$

After taking difference between squares of Eq. (79) and Eq. (80), applying the normalization condition given Eq. (71), $\left|u_{k}\right|^{2}-\left|v_{k}\right|^{2}=1$, then we obtain the excitation energy

$$
\epsilon_{k}=\sqrt{E_{k}^{2}+2 g n_{0} E_{k}}
$$

Taking a summation of Eq. (799) and Eq. (80) gives

$$
u_{k}=\frac{\left(E_{k}+\epsilon_{k}\right) v_{k}}{\epsilon_{k}-E_{k}}
$$

Applying the $\left|u_{k}\right|^{2}-\left|v_{k}\right|^{2}=1$, the Eq. (82) gives

$$
v_{k}^{2}=\frac{\left(\epsilon_{k}-E_{k}\right)^{2}}{4 E_{k} \epsilon_{k}} .
$$


From Eq. (82) and Eq. (831), we finally obtain

$$
v_{k}= \pm \frac{1}{2}\left(\sqrt{\frac{\epsilon_{k}}{E_{k}}}-\sqrt{\frac{E_{k}}{\epsilon_{k}}}\right), \quad \text { and } \quad u_{k}= \pm \frac{1}{2}\left(\sqrt{\frac{\epsilon_{k}}{E_{k}}}+\sqrt{\frac{E_{k}}{\epsilon_{k}}}\right) .
$$

Substituting Eq. (84) into the Eq. (75), and solving the summation of normal and anomalous density, then we have

$$
\left\langle\psi^{\prime \dagger} \psi^{\prime}\right\rangle+\left\langle\psi^{\prime} \psi^{\prime}\right\rangle=\frac{1}{L} \sum_{k}\left[\frac{E_{k}}{\epsilon_{k}}\left(N_{k}+\frac{1}{2}\right)-\frac{1}{2}\right] .
$$

The Eq. (86) consists quantum vacuum fluctuations and thermal fluctuations of the system. At zero temperature all particles are in the condensate, i.e. $N_{k}=0$ at $t=0$ and $N_{k} \neq 0$ for finite temperature. Therefore, density of non-condensate can be represented as

$$
\begin{aligned}
\left\langle\psi^{\prime \dagger} \psi^{\prime}\right\rangle+\left.\left\langle\psi^{\prime} \psi^{\prime}\right\rangle\right|_{\text {total }} & =\left\langle\psi^{\prime \dagger} \psi^{\prime}\right\rangle+\left.\left\langle\psi^{\prime} \psi^{\prime}\right\rangle\right|_{T=0}+\left\langle\psi^{\prime \dagger} \psi^{\prime}\right\rangle+\left.\left\langle\psi^{\prime} \psi^{\prime}\right\rangle\right|_{T \neq 0} \\
& =\frac{1}{L} \sum_{k}\left[\frac{E_{k}}{\epsilon_{k}}\left(N_{k}+1\right)-1\right] .
\end{aligned}
$$

From Eq. (68), the $M$ body correlation function at weak coupling regime is

$$
g_{M}^{w}=n^{M}\left(1+\frac{M(M-1)}{2 \pi n} \int_{-\infty}^{\infty} d k\left[\frac{E_{k}}{\epsilon_{k}}\left(N_{k}+1\right)-1\right]\right) .
$$

\section{APPENDIX C}

Consider the integral term in Eq. (32). There are $j$ points between 0 and $x$ and $(N-j)$ points between $x$ and $L$ among the $(N+2)$ particles. Here we present the calculation of the following form of the integration:

$$
\begin{aligned}
& \frac{1}{\mathcal{N}^{2}} \int \ldots \int_{\tilde{x}_{1} \leq \ldots \leq \tilde{x}_{N+2}} d x_{1} \ldots d x_{N}(j+1) \frac{\partial}{\partial x}\left|\psi_{N+2}^{F}\right|^{2} \\
= & \frac{1}{\mathcal{N}^{2}} \frac{\partial}{\partial x} \sum_{j=0}^{N} \frac{(j+1)}{j !(N-j) !} \int_{0}^{x} \ldots \int_{0}^{x} d x_{1} \ldots d x_{j} \int_{x}^{L} \ldots \int_{x}^{L} d x_{j+1} \ldots, d x_{N}\left|\psi_{N+2}^{F}\right|^{2} \\
= & \frac{1}{\mathcal{N}^{2}} \frac{1}{N !} \frac{\partial}{\partial x} \frac{\partial}{\partial \zeta} \zeta \sum_{j=0}^{N} \zeta^{j}\left(\begin{array}{c}
N \\
j
\end{array}\right) \int_{0}^{x} \ldots \int_{0}^{x} d x_{1} \ldots d x_{j} \int_{x}^{L} \ldots \int_{x}^{L} d x_{j+1} \ldots,\left.d x_{N}\left|\psi_{N+2}^{F}\right|^{2}\right|_{\zeta=1} \\
= & \left.\frac{1}{\mathcal{N}^{2}} \frac{1}{N !} \frac{\partial}{\partial x}\left[\frac{\partial}{\partial \zeta} \zeta \prod_{l=1}^{N}\left(\int_{x}^{L}+\zeta \int_{0}^{x}\right) d x_{l}\left|\psi_{N+2}^{F}\right|^{2}\right]\right|_{\zeta=1} \\
= & \left.\frac{1}{\mathcal{N}^{2}} \frac{1}{N !} \frac{\partial}{\partial x^{F}}\left[\frac{\partial}{\partial \zeta} \zeta \prod_{l=1}^{N}\left(\left[\int_{0}^{L}-\int_{0}^{x}\right]+\zeta \int_{0}^{x}\right) d x_{l}\left|\psi_{N+2}^{F}\right|^{2}\right]\right|_{\zeta=1} \\
= & \left.\frac{1}{\mathcal{N}^{2}} \frac{1}{N !} \frac{\partial}{\partial x}\left[\frac{\partial}{\partial \zeta} \zeta \prod_{l=1}^{N}\left(\int_{0}^{L}+(\zeta-1) \int_{0}^{x}\right) d x_{l}\left|\psi_{N+2}^{F}\right|^{2}\right]\right|_{\zeta=1} ^{N} \\
= & \left.\frac{1}{\mathcal{N}^{2}} \frac{1}{N !} \frac{\partial}{\partial x}\left[\prod_{l=1}^{N} \int_{0}^{L} d x_{l}\left|\psi_{N+2}^{F}\right|^{2}+\frac{\partial}{\partial \zeta} \zeta \sum_{l=0}^{N} \int_{0}^{x}(\zeta-1) d x_{l} \prod_{j=1, j \neq l}^{N} \int_{0}^{L} d x_{j}\left|\psi_{N+2}^{F}\right|^{2}\right]\right|_{\zeta=1}+O\left((\zeta-1)^{2}\right) \\
= & \left.\frac{1}{\mathcal{N}^{2}} \frac{1}{N !} \frac{\partial}{\partial x}\left[\prod_{l=1}^{N} \int_{0}^{L} d x_{l}\left|\psi_{N+2}^{F}\right|^{2}+N \frac{\partial}{\partial \zeta} \zeta(\zeta-1) \int_{0}^{x} d x_{l} \prod_{j=1, j \neq l}^{N} \int_{0}^{L} d x_{j}\left|\psi_{N+2}^{F}\right|^{2}\right]\right|_{\zeta=1} \\
= & \frac{1}{\mathcal{N}^{2}} \frac{1}{N !} \frac{\partial}{\partial x} \prod_{l=1}^{N} \int_{0}^{L} d x_{l}^{F}\left|\psi_{N+2}^{F}\right|^{2}+\frac{\partial}{\partial x} \int_{0}^{x} d x_{l} \frac{1}{\mathcal{N}^{2}} \frac{1}{(N-1) !} \prod_{j=1, j \neq l}^{L} \int_{0}^{L} d x_{j}\left|\psi_{N+2}^{F}\right|^{2} \\
= & \alpha^{N+1}\left(\frac{\partial}{\partial x} g_{2}^{N+2, F}(0, x)+\frac{\partial}{\partial x} \int_{0}^{x} d x_{l} g_{3}^{N+2, F}\left(0, x, x_{l}\right)\right),
\end{aligned}
$$


where we can write

$$
\prod_{l=1}^{N}\left(\int_{x}^{L}+\zeta \int_{0}^{x}\right) d x_{l}\left|\psi_{N}^{F}\right|^{2}=\sum_{j=0}^{N} \zeta^{j}\left(\begin{array}{c}
N \\
j
\end{array}\right) \prod_{l=1}^{N} \int_{0}^{L} d x_{l}\left|\psi_{N}^{F}\right|^{2}
$$

What below gives a further demonstration on spacial cases, see Eq. (90) and Eq. (91): Considering a system with $N=3$,

$$
\begin{aligned}
& \prod_{l=1}^{3}\left(\int_{x}^{L}+\zeta \int_{0}^{x}\right) d x_{l}\left|\psi_{3}^{F}\right|^{2} \\
= & {\left[\iiint_{x}^{L} d x_{1} d x_{2} d x_{3}+\left(\iint_{x}^{L} d x_{1} d x_{3} \zeta \int_{0}^{x} d x_{2}+\iint_{x}^{L} d x_{2} d x_{3} \zeta \int_{0}^{x} d x_{1}+\iint_{x}^{L} d x_{1} d x_{2} \zeta \int_{0}^{x} d x_{3}\right)\right.} \\
& \left.+\left(\int_{x}^{L} d x_{3} \zeta^{2} \iint_{0}^{x} d x_{1} d x_{2}+\int_{x}^{L} d x_{1} \zeta^{2} \iint_{0}^{x} d x_{2} d x_{3}+\int_{x}^{L} d x_{2} \zeta^{2} \iint_{0}^{x} d x_{1} d x_{3}\right)+\zeta^{3} \iiint_{0}^{x} d x_{1} d x_{2} d x_{3}\right]\left|\psi_{3}^{F}\right|^{2},
\end{aligned}
$$

and

$$
\begin{aligned}
& \sum_{j=0}^{3} \zeta^{j}\left(\begin{array}{c}
3 \\
j
\end{array}\right) \prod_{l=1}^{3} \int_{0, x}^{x, L} d x_{l}\left|\psi_{3}^{F}\right|^{2} \\
= & {\left[\iiint_{x}^{L} d x_{l} d x_{2} d x_{3}+\left(\zeta \int_{0}^{x} d x_{1} \iint_{x}^{L} d x_{2} d x_{3}+\zeta \int_{0}^{x} d x_{2} \iint_{x}^{L} d x_{1} d x_{3}+\zeta \int_{0}^{x} d x_{3} \iint_{x}^{L} d x_{1} d x_{2}\right)\right.} \\
& \left.+\left(\zeta^{2} \iint_{0}^{x} d x_{1} d x_{2} \int_{x}^{L} d x_{3}+\zeta^{2} \iint_{0}^{x} d x_{1} d x_{3} \int_{x}^{L} d x_{2}+\zeta^{2} \iint_{0}^{x} d x_{2} d x_{3} \int_{x}^{L} d x_{1}\right)+\zeta^{3} \iiint_{0}^{x} d x_{1} d x_{2} d x_{3}\right]\left|\psi_{3}^{F}\right|^{2} .
\end{aligned}
$$

* e-mail xwe105@wipm.ac.cn

\section{REFERENCES}

[1] Lieb E H and Liniger W 1963 Phys. Rev. Lett. 130, 1605.

[2] Yang C N and Yang C P 1969 J. Math, Phys. 10, 1115.

[3] Korepin V E, Bogoliubov N M and Izergin A G, 1993 Cambridge University Press, Cambridge, England.

[4] Jiang Y Z, Chen Y Y and Guan X W, 2015 Chin. Phys. B. 24, 050311.

[5] Paredes B, Widera A, Murg V, Mandel O, Fölling S, Cirac I, Shlyapnikov G V, Hänsch T W, and Bloch I,2004 Nature 429, 277.

[6] Kinoshita T, Wenger T and Weiss D S 2004 Science 305, 1125.

[7] Haller E, Gustavsson M, Mark M J, Danzl J G, Hart R, Pupillo G, and Nagerl H-C 2009 Science 325, 1224.

[8] Yang B, Chen Y-Y, Zheng Y-G, Sun H, Dai H-N, Guan X-W, Yuan Z-S, and Pan J-W 2017 Phys. Rev. Lett. 119 , 165701.

[9] Kinoshita T, Wenger T, and Weiss D S 2006 Nature 440, 900

[10] Hofferberth S, Lesanovsky I, Fischer B, Schumm T and Schmiedmayer J 2007 Nature 449, 324.

[11] Astrakharchik G E and Giorgini S, 2006 J. Phys. B: At. Mol. Opt. Phys. 39, S1.

[12] Astrakharchik G E and Giorgini S 2003 Phys. Rev. A 68, 031602(R).

[13] Gangardt D M and Shlyapnikov G V 2003 New J. Phys. 5, 79.

[14] Kheruntsyan K V, Gangardt D M, Drummond P D and Shlyapnikov G V 2003 Phys.Rev. Lett. 91, 040403.

[15] Gangardt D M and Shlyapnikov G V 2003 Phy.Rev.Lett. 90, 010401.

[16] Kinoshita T, Wenger T and Weiss D. S. 2005 Phys.Rev.Lett. 95, 190406.

[17] Kormos M, Mussardo G, and Trombettoni A, 2010 Phys. Rev. A 81, 043606;

[18] Kormos M,ChouY Z and Imambekov A, 2011 Phys.Rev.Lett. 107, 230405.

[19] Nandani E J K P, Romer R A, Tan S and Guan X W 2016 New J. Phys. 18, 055014. 
[20] Caux J S 2009 J. Math. Phys. 50, 095214.

[21] Caux J S and Calabrese P 2006 Phys. Rev. A 74, 031605.

[22] Panfil M and Caux J S 2014 Phys. Rev. A 89, 033605.

[23] Girardeau M 1960 J. Math. Phys. 1, 516.

[24] Cheon T and Shigehara T 1999 Phys. Rev. Lett. 82, 2536.

[25] Cherny A Y and Brand J 2006 Phys. Rev. A. 73, 023612.

[26] Lenard A 1966 J. Math. Phys. 7, 1268.

[27] Lenard A 1964 J. Math. Phys. 5, 930.

[28] Caux J S, Calabrese P and Slavnov N A 2007 J. Stat. Mech. P01008.

[29] Forrester P J, Frankel N E, Garoni T M and Witte N S 2003 Phys. Rev. A 67, 043607.

[30] Forrester P J , Frankel N E and Makin M I 2006 Phys. Rev. A 74, 043614.

[31] Jimbo M and Miwa T 1981 Phys. Rev. D 24, 3169.

[32] Santachiara R and Calabrase P 2008 J. Stat. Mech. P06005.

[33] Vaidya H G and Tracy C A, 1979 Phys. Rev. Lett.42, 3.

[34] Jimbo M, Miwa T, Mori Y and Sato M, 1980 Physica D 1, 80.

[35] Vaidya H G and Tracy C A 1979 J. Maths. Phys.20, 2291.

[36] Olshani M and Dunjko V 2003 New J. Phys 5, 98.

[37] Olshani M and Dunjko V 2003 Phys Rev Lett 91, 090401.

[38] Olshani M, Dunjko V, Minguzzi A, and Lang G, 2017 Phys. Rev. A 96, 033624.

[39] Tan S 2008 Ann. Phys. 323, 2952.

[40] Tan S 2008 Ann. Phys. 323, 2987.

[41] Tan S 2008 Ann. Phys. 323, 2971.

[42] Barfknecht R E, Brouzos I and Foerster A 2015 Phys. Lett. A 91, 043640.

[43] Zhang S and Leggett A J 2009 Phys. Rev. A 79, 023601.

[44] Werner F, Tarruell L and Castin Y 2009 Eur. Phys. J. B 68, 401.

[45] Braaten E and Platter L 2009 Laser Physics 19, 550.

[46] Pâtu O I and Klümper A 2017, Phys. Rev. A 96, 063612.

[47] Drut J E, Lahde TA and Ten T 2011 Phys. Rev. Lett 106, 205302.

[48] Barth M and Zwerger W 2011 Ann. Phys. 326, 2544.

[49] Yu Z, Thywissen J H and Zhang S 2015 Phys. Rev. Lett. 115, 135304.

[50] Yoshida S M and Ueda M 2015 Phys. Rev. Lett. 115, 135303.

[51] Cui X 2016 Phys. Rev. A 94, 043636.

[52] Sekino Y, Tan S and Nishidha Y 2018 Phys. Rev. A 97, 013621.

[53] Minguzzi A, Vignolo P and Tosi M P 2002 Phys. Lett. A 294, 222.

[54] Wei Xu and Rigol M 2015 Phys. Rev. A 92, 063623.

[55] Wild R J, Makotyn P, Pino J M, Cornell E A and Jin D S 2012 Phys. Rev. Lett 108, 145305.

[56] Yin X, Guan X W, Zhang Y, Su H and Zhang S, 2018 arXiv:1803.05119.

[57] Guan X W 2014 Int. J. of Mod. Phys. B 28, 1430015.

[58] Fabbri N, Panfil M, Clement D, Fallani L, Inguscio M, Fort C and Caux J S 2015 Phys. Rev. A 91, 043617.

[59] Brand J and Cherny A Y 2005 Phys. Rev. A. 72, 033619.

[60] Wu Y S 1994 Phys. Rev. Lett. 73, 922.

[61] Bernard D and Y S Wu 1994 cond-mat/9404025v2.

[62] Ha Z N C 1994 Phys. Rev. Lett. 73, 1574.

[63] Murthy M V N and Shankar R, 1994 Phys. Rev. Lett. 73, 3331.

[64] Ha Z N C, 1995 Nucl. Phys. B 435, 604.

[65] Batchelor M T, Guan X W, and Oelkers N 2006 Phys. Rev. Lett. 96, 210402.

[66] Batchelor B T and Guan X-W 2007 Laser Phys. Lett. 4, 77.

[67] Mehta M L 1970 Random matrices-3rd, Elsever academy press.

[68] Pâtu O I and Klümper A 2017 Phys. Rev. A 96, 063612. 